\title{
Does Plasticity Promote Criticality?
}

\author{
Filipe Peliz Pinto Teixeira and Murray Shanahan
}

\begin{abstract}
Neuronal avalanches are a cortical phenomenon characterised by bursts of activity bracketed by periods of quiescence. It has been shown both in vivo and in vitro that the size and length of avalanche events conform to power law-like distributions, suggesting the system is within or near a critical state. This work investigates the interplay of network connectivity, synaptic plasticity, and criticality. Using two different network construction algorithms, we demonstrate that Spike Timing Dependent Plasticity (STDP) robustly drives the network towards a critical state. Our findings show that, while the initial distribution of synaptic weights plays a significant role in attaining criticality, the network's topology at the local level has little or no impact.
\end{abstract}

\section{INTRODUCTION}

$\mathrm{C}$ ORTICAL networks demonstrate a variety of phenomena which have been hypothesized to underlie cognition. Examples include synchronous oscillations [1], [2], modular firing [3], and neuronal avalanches [4], [5]. Various factors, such as excitatory and inhibitory neural activity as well network connectivity impact these phenomena. This paper focuses on the impact of network structure on neuronal avalanches and the identification of criticality. Neuronal avalanches are bursts of activity followed by episodes of quiescence. It has been shown in vivo [6] that neuronal avalanches have statistical properties reminiscent of critical systems [4].

Using two parametrised algorithms for synthesising networks we constructed 3000 different structures which underwent the same training. Using Spike Timing Dependent Plasticity (STDP) we evolved all networks towards a specific dynamical regime dependent on initial excitatory and inhibitory synaptic strength. After one minute of training, we stimulated the network and measured the resulting avalanche activity for two minutes.

By measuring small-worldness [7], modularity [3], and synaptic weight distributions we analyse the post learning networks and demonstrate how network structure impacts avalanche activity. We show that power

Filipe Peliz Pinto Teixeira is with the Department of Computing, Imperial College, London, United Kingdom (email: fp10@imperial.ac.uk).

Murray Shanahan is with the Department of Computing, Imperial College, London, United Kingdom (email: m.shanahan@imperial.ac.uk).

This work is supported by the Commonwealth Doctoral Scholarship ZACS-2013-547. law avalanche (or rather more appropriately power law-like) distributions arise for many post learning networks, therefore demonstrating how STDP may be a sufficient process for evolving towards a critical state. These findings expand on $\mathrm{Li}[8]$ and De Arcangelis[9] who originally show that a Hebbian learning process is sufficient for achieving critical states in simple network models.

This paper begins by defining criticality and describing the various approaches for identifying critical systems. We describe how avalanches play a role in identifying criticality and why we use this approach. Following the introduction to criticality, we define the neural network model as well as the training environment. The paper concludes by showing how network structure impacts avalanche activity and by demonstrating that STDP is a sufficient process to produce power law-like avalanche distributions.

\section{BACKGROUND}

Neuronal avalanche activity was originally observed in vitro by Beggs and Plenz [4] and later in vivo by Gireesh [6] and Petermann [10]. Beggs and Plenz identify avalanches by first dividing neural activity into a series of continuous windows of size $\Delta t=4 \mathrm{~ms}$. A window is active if any neurons fire, otherwise it is inactive. An avalanche is a continuous series of active windows encapsulated by inactive windows. They then formally quantify avalanches by size (number of neural firings within avalanche) and length (number of continuous active windows).

When measuring the size and length distributions of neuronal avalanches Beggs and Plenz show that they conform to a power law-like distribution $\left(P(n) \sim n^{-\alpha}\right)$ of exponents -1.5 and -2 respectively. Identifying a power law indicates the system is in, or near a critical state [11], [12], [13]. Being in a critical state represents the system being poised between order and disorder as well as the possibility of a minor event triggering a chain reaction leading to a large response [11]. We observe this behaviour in natural events such as forest fires, floods, and earthquakes. Natural systems (including cortical networks) self organize into this state via a critical branching process - Self Organized Criticality [11], [14], [4].

It has been argued that this process is not just a coincidence [11], [5], [12]. By self-organizing via neuronal avalanche activity to operate near criticality, the brain is said to optimize network behaviour. Beggs 
claims that this phenomenon affects network behaviour with regards to information processing, information storage, computational power, and stability - The Criticality Hypotheses [15], [16]. Shew [5] extends this hypothesis by proposing that a network near criticality maximizes dynamic range.

In addition to recent work focusing on the impacts of criticality, we also see many authors aiming to better understand the phenomenon at the local [8], [9] and global level [17]. In a similar vein, this paper aims to improve our understanding of neuronal avalanches and their contribution to criticality by modelling complex local network structures and analysing how their connectivity contributes to the formation of avalanches.

\section{Methodology}

\section{A. Neural Network Model}

Continuing from the work of [8], [18], [9], we construct a novel neural network reproducing avalanches. The behaviour of a single neuron is produced using the Izhikevich model:

$$
\begin{aligned}
& \begin{array}{l}
v^{\prime}=0.04 v^{2}+5 v+140-u+I \\
u^{\prime}=a(b v-u)
\end{array} \\
& \text { if } v \geq 30 \mathrm{mV}, \text { then }\left\{\begin{array}{l}
v \leftarrow c \\
u \leftarrow u+d
\end{array}\right.
\end{aligned}
$$

where $v$ and $u$ represent the neuron's membrane potential and recovery respectively. The parameters $a, b, c$, and $d$ are set to model either excitatory $\left(0.02,0.2,-65+15 r^{2}, 8-6 r^{2}\right)$ or inhibitory $(0.02+$ $0.08 r, 0.2-0.05 r,-65,2)$ neurons. The variable $r$ is drawn from a uniform distribution $U(0,1)$ to introduce some variability in the neuronal population. $I$ represents current from neighbouring neurons as well as external stimulation which we use to interact with the system. When the membrane potential $v$ exceeds a threshold, eq (2) models the after-spike reset behaviour. This approach is computationally efficient and comparable to neural behaviour in nature [19].

Our model uses 800 excitatory neurons and 200 inhibitory neurons. Synaptic delays from inhibitory neurons are $1 \mathrm{~ms}$ and from excitatory neurons are random $U(1,20)$. Inhibitory weights are fixed at $w_{i j}=4$ and the pre-learning excitatory weight is a parameter depending on network connectivity and the dynamical regime the network drives towards (discussed later).

We define connectivity through two techniques Watts-Strogatz [7] and Klemm-Eguiluz [20]. Both use wiring control parameters $p \in[0,1]$, allowing us to produce more diverse networks. The Watts-Strogatz procedure is well known so we omit further discussion on their technique. However, the approach by KlemmEguiluz is comparatively under utilised, thus we discuss their method further.

The Klemm-Eguiluz procedure produces networks of modularity and small worldness comparable to the Watts-Strogatz approach. However, unlike WattsStrogatz, the degree distribution is mathematically defined (i.e. power law distribution). Furthermore, Klemm-Eguiluz produces networks with more richly connected neurons and hub nodes. This is achieved by maintaining an active list of neurons which are more likely to receive connections from other neurons - preferential attachment.

We modify their approach so the wiring parameter $p$ does not impact the number of synapses. Our modified version of their approach is given in algorithm 1 . Prettejohn provides a comparative and detailed review of both Klemm-Eguiluz and Watts-Strogatz networks [21].

Watts-Strogatz networks are built with $k=10$ degree, Klemm-Eguiluz with $m=10$ active nodes and $\kappa=0.5$ directional probability. An important feature of the Watts-Strogatz approach and our version of the Klemm-Eguiluz algorithm is that the value of the wiring probability $p$ does not affect the number of synapses. This allows us to understand how only network connectivity influences the formation of neuronal avalanches.

A complex neural structure alone is insufficient for producing neuronal avalanches. Dynamic long range neuronal avalanches are unlikely to form with a flat synaptic weight distribution. Using Spike Timing Dependant Plasticity (STDP) [22] allows for a more complex weight distribution to form over time. The following STDP function modifies excitatory synapses based on timing between spikes:

$$
\Delta w=\left\{\begin{array}{l}
A_{+} e^{-\Delta / \tau_{+}} \text {if } \Delta t \geq 0 \\
-A_{-} e^{\Delta / \tau_{-}} \text {if } \Delta t<0
\end{array}\right.
$$

where $\Delta w$ is the weight change, $\Delta t=t_{j}-t_{i}$ is the time difference between the presynaptic $i$ and postsynaptic $j$ spike. $\tau_{+}$and $\tau_{-}$define the temporal window of the synaptic modifications, while $A_{+}$and $A_{-}$define the strength of the modifications. We use a temporal window of $\tau_{+}=\tau_{-}=20$ with modification strengths of $A_{+}=0.05$ and $A_{-} / A_{+}=1.05$ as used by [8] and originally defined by [22].

Our STDP process is active until synaptic modification begins to stagnate, at which point STDP is turned off to prevent over pruning. We use the initial excitatory weight to guide the formation of neuronal avalanches towards sub-critical, critical, or super-critical regimes. Weights of $W S_{w}=(5,9,11)$ and $K E_{w}=(11,15,17)$ guide Watts-Strogatz and Klemm-Eguiluz networks respectively towards either a sub-critical, critical or super-critical regime. A genetic 


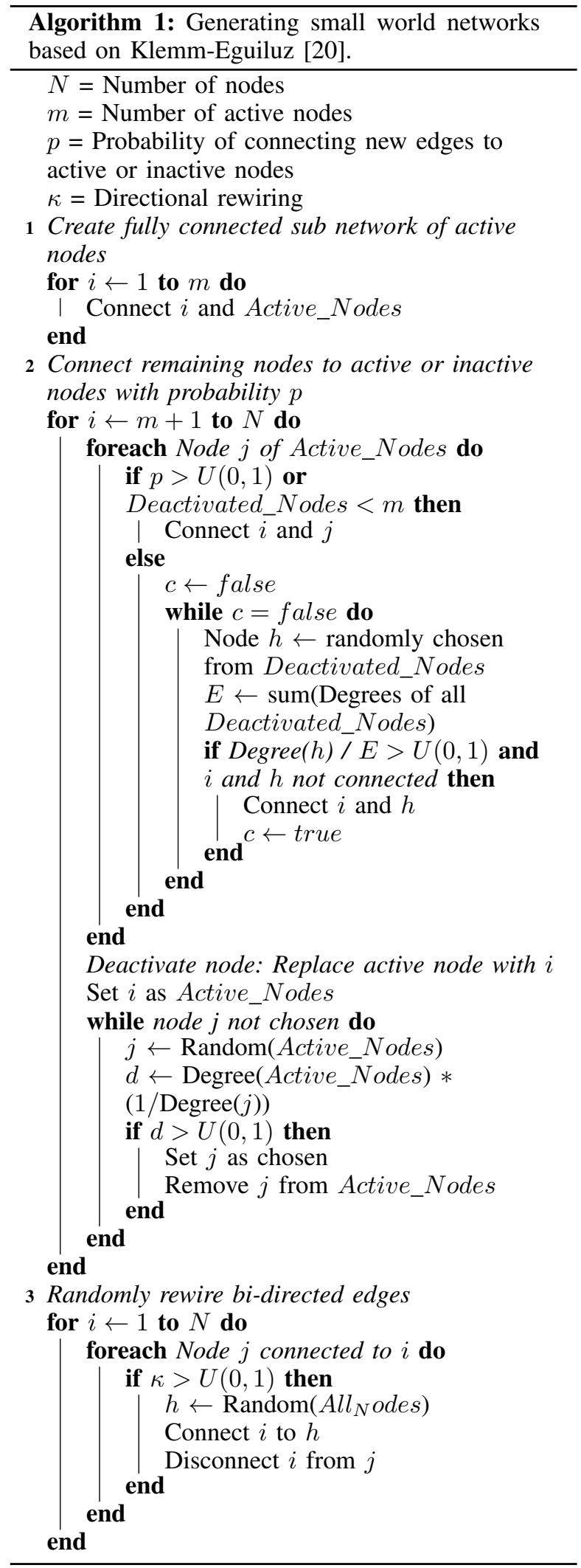

algorithm was used to identify the ideal weights resulting in critical activity $\left(W S_{w}=9\right.$ and $\left.K E_{w}=15\right)$. By choosing smaller and larger values we further analyse sub-critical and super-critical regimes. Different values are used due to each technique producing networks with different numbers of synapses 10,000 and 19,890 for Watts-Strogatz and Klemm-Eguiluz respectively.

The model enables us to produce a variety of avalanche dynamics under different circumstances. This aids us in better understanding how network structure impacts the formation of neuronal avalanches.

\section{B. Network Measures}

This section describes methods taken from graph theory for measuring attributes common of complex networks. Measuring such features aids in establishing a correlation between specific network characteristics and critical dynamics.

A common feature of complex networks is high clustering [23]: If node $x$ is connected to $y$ and $z$ then it is likely that $y$ and $z$ are connected. For example, your friends are likely to know each other. For our purposes nodes with only one neighbour - leaf nodes - have a clustering coefficient of zero, other authors may use one for such cases. Kaiser [23] discusses the effects of using zero or one in greater detail.

A complex network is further defined by a short average path length [24], [7]. Both these features are typical of small world networks.

Formally, we define small world networks by:

$$
\sigma_{G}=\frac{\gamma_{G} / \gamma_{\text {rand }}}{\lambda_{G} / \lambda_{\text {rand }}}
$$

where $\gamma_{G}$ and $\gamma_{\text {rand }}$ are the clustering coefficients of network $G$ and an equivalent random network respectively; and $\lambda_{G}$ and $\lambda_{\text {rand }}$ are the mean path lengths of network $G$ and an equivalent random network respectively.

The clustering coefficient of a node $j$ is the fraction of the set of all possible edges between immediate neighbours of $j$ that are actual edges. The average clustering coefficient $\gamma_{G}$ is this value averaged for all nodes in the network $G$. The mean path length $\lambda_{G}$ is the minimum number of steps from node $i$ to $j$ averaged for all node pairs in network $G$. The clustering coefficient of a random network with $n$ nodes and average degree $k$ is $\gamma_{\text {rand }}=k / n$ while the mean path length is $\lambda_{\text {rand }}=\ln (n) / \ln (k)$ [7].

We further define a complex network by its modularity. A network is modular if nodes can be partitioned into populations which are highly intra-connected and sparsely inter-connected [3]. Newman [3] formally defines modularity with respect to a given partitioning $c$ :

$$
Q_{G}=\frac{1}{2 m} \sum_{i, j}\left(G_{i j}-\frac{k_{i} k_{j}}{2 m}\right) \delta_{c_{i} c_{j}}
$$


where $G_{i j}>0$ indicates the presence of a connection between $i$ and $j, m$ is the number of edges in the network, $c_{x}$ indicates the module of neuron $x$, and

$$
\delta_{x y}= \begin{cases}1 & \text { if } x=y \\ 0 & \text { otherwise }\end{cases}
$$

Network modularity $Q_{G}$ has a maximum value of one and is negative when there are fewer connections within a module than between modules. When generating networks probabilistically their modular division is unknown. Using the Brain Connectivity Toolbox by [25] we heuristically find network modules. However the algorithm of [25] returns varying results on different attempts. To control for variance we run modularity analysis 100 times, and pick the community division resulting in the highest network modularity $Q_{G}$.

Only post-learning networks produce dynamic long range avalanches. Pre- and post-learning networks differ slightly in connectivity but primarily in synaptic strengths. Due to this, in addition to computing the modularity and small-worldness, we further analyse networks according to their weight distribution - the synaptic strengths from excitatory neurons to all other neurons. This approach provides a more detailed look at each network's synaptic structure

With these network measures we demonstrate the correlation between network structure and avalanche activity. We also show how networks evolve from a pre-learning state to a post-learning dynamical regime.

\section{Experiments}

We ran 3000 simulations; 1000 for each dynamical regime (sub-critical, critical, and super-critical) and within each regime, 500 for each network construction technique (Watts-Strogatz and Klemm-Eguiluz). The simulations were hardware-accelerated using a GPU in combination with the NeMo framework [26]. Each simulation entails:

1) Generating the network structure for a given wiring probability $p$.

2) Training the network for 30 seconds with an external current $I=6$ to excitatory neurons.

3) Generating neuronal activity for two minutes with a lower external current $I=3$ to excitatory neurons.

4) Measuring pre and post learning network statistics.

5) Measuring avalanche sizes according to windows of $\Delta t=1 \mathrm{~ms}$ (Due to a larger model we use a smaller time scale in comparison to [4], [8]).

6) Fitting and measuring confidence of power law distributions according to [27] and [13].

\section{RESULTS}

We use the terms sub-critical, critical, and supercritical when referring to neuronal activity. Figure 1 shows what these terms refer to from the perspective of neural firings. Subcritical firing reveals no obvious pattern and is interpreted as noise, while super-critical firing reveals an obvious banding due all neurons constantly firing together in large avalanches. On the other hand, critical firing falls between these two regimes; we see some banding activity occurring but often breaking down due to larger avalanches being less likely. If sub-critical firing is noise and super-critical is overly ordered then a critical network is likely to show more interesting dynamics.

Similarly to [4], [8], when we measure these firings

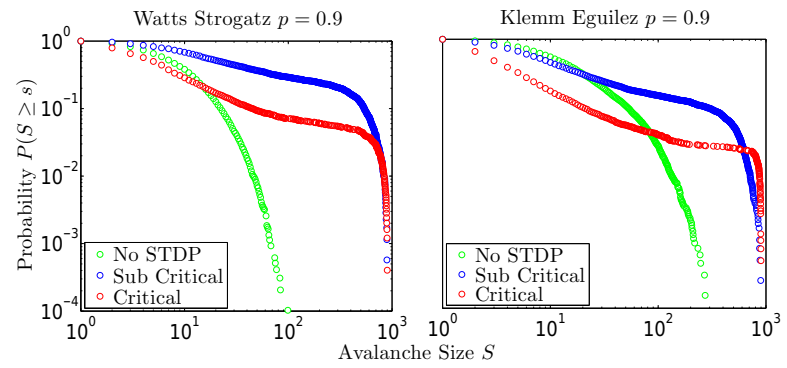

Fig. 2: Power law-like probability distributions $P(S \geq$ $s$ ) for both example Watt-Strogatz and Klemm-Eguiluz networks.

we reveal power law-like distributions for the size and length of avalanches. Our plotted distributions show the probability that the avalanhce size is less-than-or-equal to $S$ rather than exactly $S$. If we plot the equivalent $P(S=s)$ the slope would appear steeper. Regardless of appearence all distributions are fit with a power law $\left(P(n) \sim n^{-\alpha}\right)$. Figure 2 shows the distributions for both Watts-Strogatz and Klemm-Eguiluz networks. The difference between untrained networks and networks within a dynamical regime is later shown in figure 5.

Low initial excitatory weights $\left(W S_{w}=5, K E_{w}=\right.$ 11) result in STDP modifying networks to exhibit a more diverse avalanche distribution, however no large avalanches occur, possibly reflecting the inability of distant neurons to communicate. High initial excitatory weights $\left(W S_{w}=11, K E_{w}=17\right)$ result in larger avalanches being more likely occur. This means distant neurons are communicating more. However, if the same neurons are in constant communication this represents the network being overly ordered and possibly less dynamic. Fine-tuned excitatory weights $\left(W S_{w}=\right.$ $\left.9, K E_{w}=15\right)$ lead to power law-like avalanche distributions with exponents -1.49 and -1.51 for WattsStrogatz and Klemm-Eguiluz respectively. We identify power laws using methods from [27], [13] which are represented by a straight line on a log-log plot (figure 


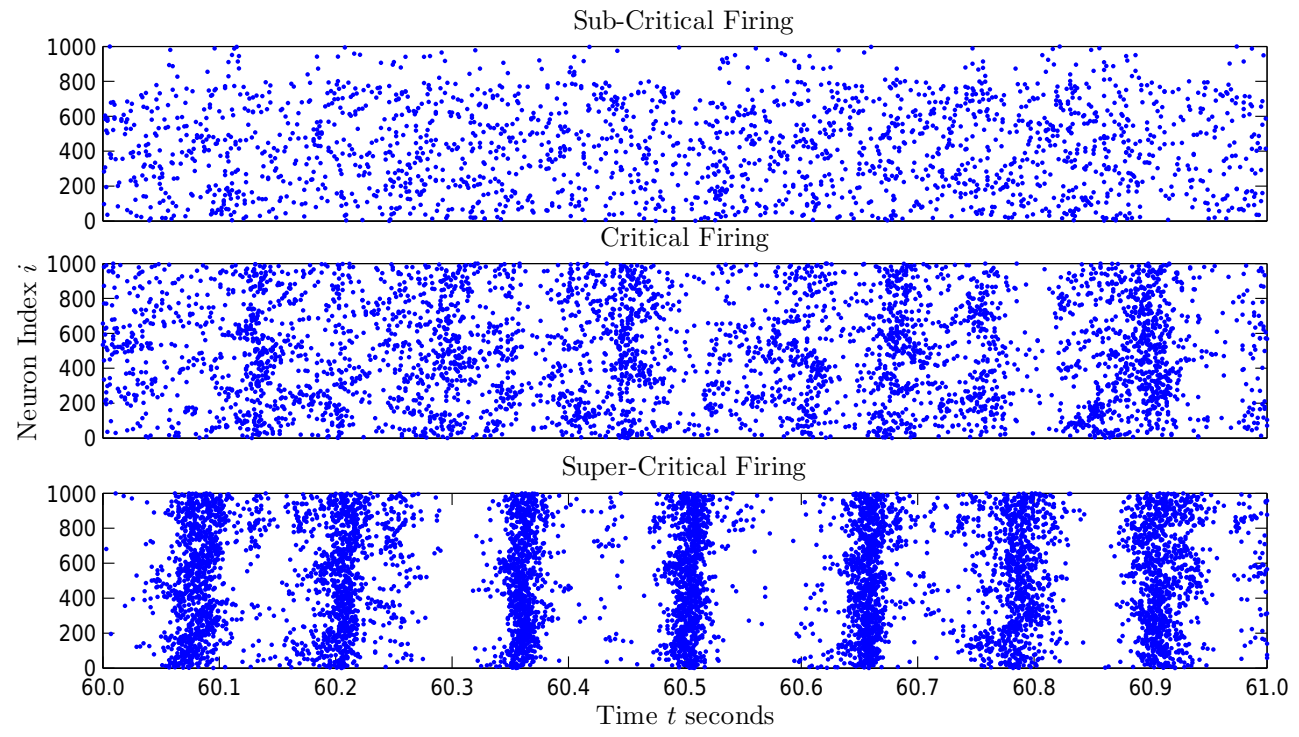

Fig. 1: Examples sub-critical, critical, and super-critical neuronal firing for 1 second. Neurons 1 - 800 are excitatory and $801-1000$ inhibitory

2). These findings agree with previous experimental results [4], [6], [10] and reflect the ability of distant neurons to communicate but not become stuck in the same "conversation".

Figures 1 and 2 mainly represent the model's
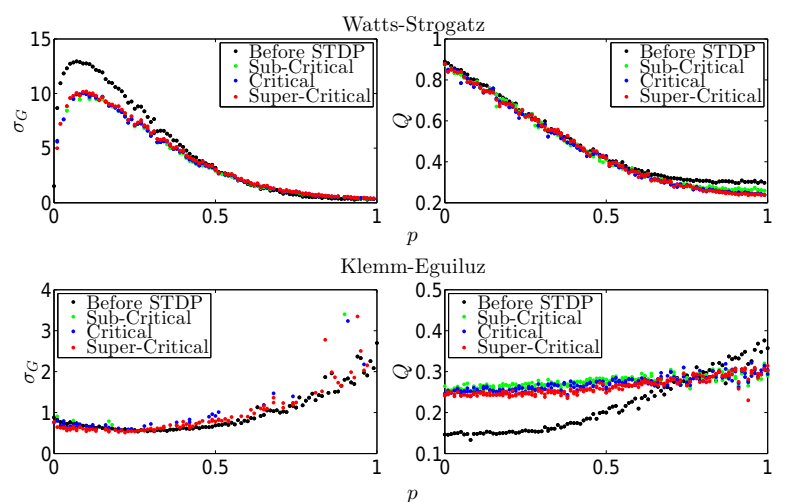

Fig. 3: Small-worldness $\sigma_{G}$ and modularity $Q$ for subcritical, critical, and super-critical networks.

ability to produce neuronal avalanches comparable to previous results [4], [8], [9]. We now question what type of local network connectivity promotes criticality. Figure 3 shows the small-worldness and modularity of sub-critial, critical, and supercritical networks for both Watts-Strogatz and Klemm-Eguilez networks for any wiring probability $p$. These results show that: 1 . Klemm-Eguiluz networks tend towards a modularity of 0.28 regardless of the resulting dynamical regime and
2. Small-worldness and modularity are not required for neural firing to follow any of these particular dynamical regimes.

Since small-worldness and modularity do not ap-
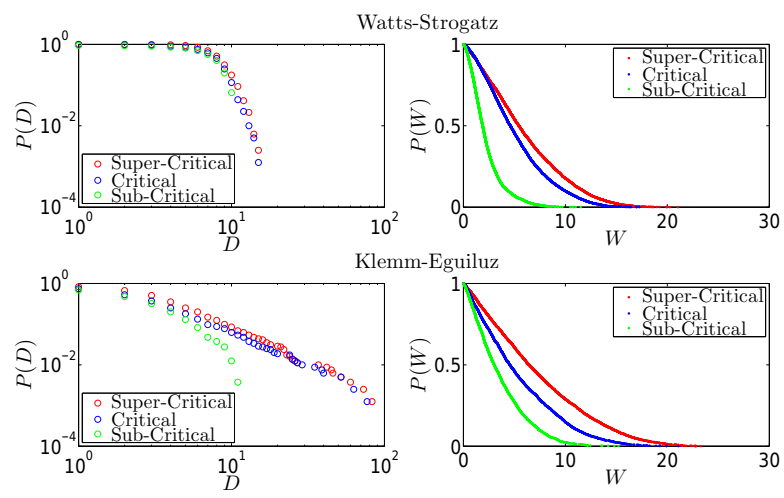

Fig. 4: Degree $D$ and weight $W$ probability distributions for sub-critical, critical, and super-critical networks

pear to affect avalanches, we carried out a more detailed analysis of sub-critical, critical, and super-critical networks for both Watt-Strogatz and Klemm-Eguiluz. When looking at the degree and weight distribution (figure 4) we see a clear difference between each regime for both network types. Both network types have different degree distributions, Watts-Strogatz being not well defined and Klemm-Eguiluz following a power law. Regardless of degree distributions both 

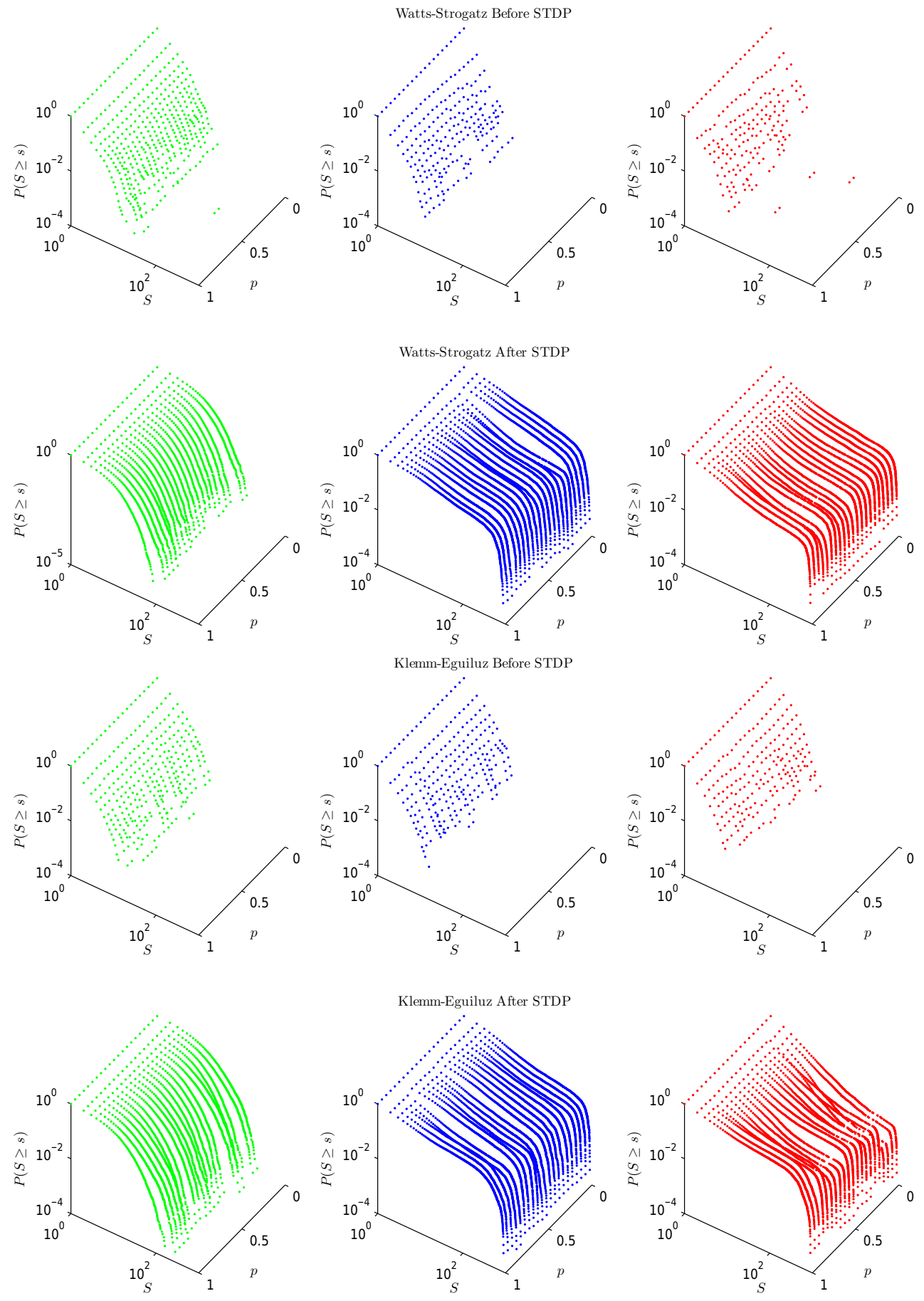

Fig. 5: Avalanche size distributions $S$ showing effect of STDP for sub-critical (green), critical (blue), and supercritical (red) networks defined by the Watts-Strogatz and Klemm-Eguiluz procedures with wiring parameter $p$ 
networks access all dynamical regimes.

We see that weight distribution clearly affects which dynamical regime the network operates within. It appears that the magnitude of the weight distribution defines the formation of avalanches and consequently the networks' criticality. From these results we hypothesise that neuronal avalanches are defined primarily by the network's weight distribution, and other factors such as small-worldness and modularity at least at the local level do not affect avalanches. However, this does not answer our initial question, does STDP promote criticality?

To answer this question we sweep the WattStrogatz and Klemm-Eguiluz wiring parameter $p$ between 0 and 1 for the three dynamical regimes. Figure 5 shows the resulting avalanche size distributions for the different values of $p$. We see that the dynamical regime towards which a network is trained by STDP is not affected by the value of $p$. Previously we saw that connectivity alone does not impact criticality, but rather the initial weight assignment affects criticality. Here we see a similar phenomenon. Initial connectivity has little influence. Rather, STDP robustly drives the network into one of the three dynamical regimes, and which regime this is depends chiefly on the initial balance between excitatory and inhibitory weights. But how robust is the learning towards critical networks specifically?

Figure 6 shows that when we ran the simulation

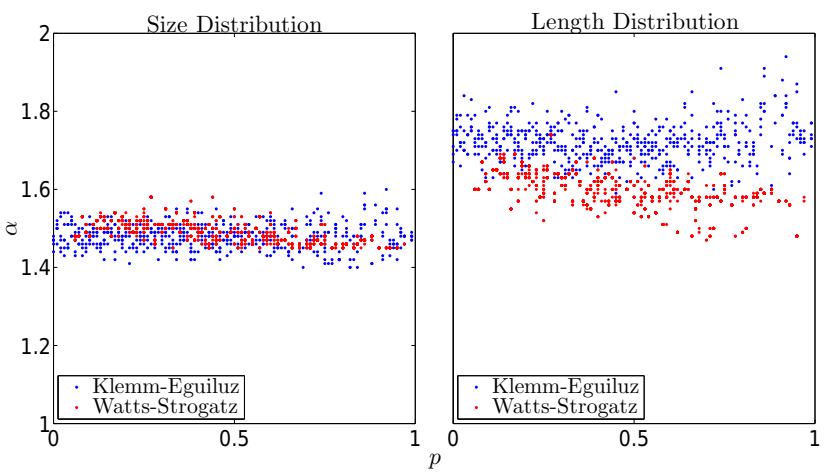

Fig. 6: Exponents $\alpha$ of avalanche size $P(S) \sim S^{-\alpha}$ and length $P(L) \sim L^{-\alpha}$ probability distributions for critical networks between wiring probability $0 \leq p \leq 1$.

500 times for each network type the resulting size exponent is the same $\approx-1.5$ with some variance $\approx 0.1$. For the length distribution we see that WattsStrogatz and Klemm-Eguiluz networks differ with exponents of $\approx-1.6$ and $\approx-1.7$ respectively. For 500 simulations we only saw 21 and 30 failures to train towards a critical network for Watts-Strogatz and Klemm-Eguiluz respectively, where a failure is defined as the confidence of the power law fit dropping below
0.1 This shows that STDP will consistently drive the size of avalanches towards the same power law-like behaviour and possibly towards criticality.

\section{Discussion}

Our work shows that STDP is a robust training process for driving a variety of networks towards the generation of avalanches whose sizes conform to a power law-like distribution. The specific dynamical regime that results is primarily dependent on the initial synaptic weight distribution. In our work, by modifying the networks' internal excitation we demonstrate how various dynamical regimes may be reached regardless of initial network connectivity. Our work not only demonstrates the power of an STDP training process towards these dynamical regimes but also demonstrates how criticality is a robust phenomenon which may be identified for a variety of networks using power lawlike avalanche distributions as indicators.

Throughout this paper we have used the term power law-like. We use this term due to the current debate regarding the validity of the power laws being identified in critical systems. Power laws are visually similar to other distributions (example gamma and exponential distributions) resulting in their often being misidentified, thus calling into question the claim of neural systems being critical. Currently many authors debate both these questions with no clear consensus so far [27], [28], [12], [29], [13].

If power laws are possibly unreliable indications of criticality, how can we confirm that neural systems are critical? An alternative method is to determine if the system exhibits a phase transition. A phase transition takes place if, while sweeping some control parameter, a sharp transition occurs in a corresponding order parameter. The Ising Model [30] exhibits this behaviour - as the temperature (control parameter) is applied, the correlation length (order parameter) between iron particles sharply maximizes for a critical temperature range. Recent work by [17] demonstrates neural phase transitions at large scale. However, at the local level, how to identify neural phase transitions as well as how the control and order parameters are selected is still largely unknown.

With no conclusion regarding the validity of the power law in these systems, and with local neural phase transitions still in need of investigation, the question of neural criticality and how to identify it remains open. Thus we have adopted the term power law-like, and following [4], [12] and [8] we assume power law-like avalanches with an exponential cut-off represent the ability for dynamic long-range correlations to form, thus showing the system may be near a critical state.

We hope to expand our work by attempting to identify local neural phase transitions, thus confirming that 
our model is in a critical state rather than simply near one. Furthermore we aim to improve our model with the inclusion of STDP affecting inhibitory synapses and with STDP being a continuous process. We aim to demonstrate that local network connectivity does not affect criticality and that criticality is a fundamental property underlying other neural phenomena such as synchronisation [1] and metastability [31].

\section{REFERENCES}

[1] P. Fries, "Neuronal gamma-band synchronization as a fundamental process in cortical computation.," Annual Review of Neuroscience, vol. 32, no. March, pp. 209-224, 2009.

[2] C. Börgers and N. Kopell, "Synchronization in networks of excitatory and inhibitory neurons with sparse, random connectivity.," Neural Computation, vol. 15, no. 3, pp. 509538, 2003.

[3] M. E. J. Newman and M. Girvan, "Finding and evaluating community structure in networks," Physical Review E - Statistical, Nonlinear and Soft Matter Physics, vol. 69, no. $2 \mathrm{Pt}$ 2, p. 16, 2003.

[4] J. M. Beggs and D. Plenz, "Neuronal avalanches in neocortical circuits.," Journal of Neuroscience, vol. 23, no. 35, pp. $11167-$ 11177, 2003.

[5] W. L. Shew, H. Yang, T. Petermann, R. Roy, and D. Plenz, "Neuronal avalanches imply maximum dynamic range in cortical networks at criticality.," Journal of Neuroscience, vol. 29, no. 49 , pp. $15595-15600,2009$.

[6] E. D. Gireesh and D. Plenz, "Neuronal avalanches organize as nested theta- and beta/gamma-oscillations during development of cortical layer 2/3," Proceedings of the National Academy of Sciences of the United States of America, vol. 105, no. 21, pp. 7576-7581, 2008.

[7] D. J. Watts and S. H. Strogatz, "Collective dynamics of 'smallworld' networks.", Nature, vol. 393, no. 6684, pp. 440-2, 1998.

[8] X. Li and M. Small, "Neuronal avalanches of a selforganized neural network with active-neuron-dominant structure.," Chaos (Woodbury, N.Y.), vol. 22, p. 023104, June 2012.

[9] L. De Arcangelis and H. J. Herrmann, "Activity-Dependent Neuronal Model on Complex Networks," Frontiers in Physiology, vol. 3, no. March, pp. 1-9, 2012.

[10] T. Petermann, T. C. Thiagarajan, M. A. Lebedev, M. A. L. Nicolelis, D. R. Chialvo, and D. Plenz, "Spontaneous cortical activity in awake monkeys composed of neuronal avalanches.," Proceedings of the National Academy of Sciences of the United States of America, vol. 106, no. 37, pp. 15921-15926, 2009.

[11] P. Bak and K. Chen, "Self-organized criticality," Scientific American; (United States), vol. 264:1, Jan. 1991.

[12] J. M. Beggs and N. Timme, "Being critical of criticality in the brain.," Frontiers in physiology, vol. 3, p. 163, Jan. 2012.

[13] J. Alstott, E. Bullmore, and D. Plenz, "powerlaw: a Python package for analysis of heavy-tailed distributions," PLOS ONE, vol. 9, p. e85777, May 2014.

[14] C. Haldeman and J. M. Beggs, "Critical branching captures activity in living neural networks and maximizes the number of metastable States.," Physical Review Letters, vol. 94, no. 5, p. 058101, 2005.
[15] J. M. Beggs, "The criticality hypothesis: how local cortical networks might optimize information processing.," Philosophical Transactions of the Royal Society - Series A: Mathematical, Physical and Engineering Sciences, vol. 366, no. 1864, pp. 329-343, 2008.

[16] D. R. Chialvo, "Emergent complex neural dynamics," Nature Physics, vol. 6, no. 10, pp. 744-750, 2010.

[17] R. Barbieri and M. Shimono, "Criticality in large-scale brain fMRI dynamics unveiled by a novel point process analysis," Networking of Psychophysics, Psychology and Neurophysiology, p. 61, 2012.

[18] L. De Arcangelis, C. Perrone-Capano, and H. J. Herrmann, "Self-organized criticality model for brain plasticity.," Physical Review Letters, vol. 96, no. 2, p. 4, 2006.

[19] E. M. Izhikevich, "Which model to use for cortical spiking neurons?," IEEE Transactions on Neural Networks, vol. 15, no. 5, pp. 1063-1070, 2004.

[20] K. Klemm and V. M. Eguíluz, "Growing scale-free networks with small-world behavior.," Physical Review E - Statistical, Nonlinear and Soft Matter Physics, vol. 65, no. 5 Pt 2, p. 057102, 2002.

[21] B. J. Prettejohn, M. J. Berryman, and M. D. McDonnell, "Methods for generating complex networks with selected structural properties for simulations: a review and tutorial for neuroscientists.," Frontiers in computational neuroscience, vol. 5, p. 11, Jan. 2011.

[22] S. Song, K. D. Miller, and L. F. Abbott, "Competitive Hebbian learning through spike-timing-dependent synaptic plasticity.," Nature neuroscience, vol. 3, pp. 919-26, Sept. 2000.

[23] M. Kaiser, "Mean clustering coefficients: the role of isolated nodes and leafs on clustering measures for small-world networks," New Journal of Physics, vol. 10, no. 8, p. 083042, 2008.

[24] J. Kleinberg, "The Small-World Phenomenon : An Algorithmic Perspective," Proceedings of the 32nd ACM Symposium on Theory of Computing, vol. 32, no. 99-1776, pp. 163-170, 2000.

[25] M. Rubinov and O. Sporns, "Complex network measures of brain connectivity: uses and interpretations.," NeuroImage, vol. 52, pp. 1059-69, Sept. 2010.

[26] A. K. Fidjeland and M. P. Shanahan, "Accelerated simulation of spiking neural networks using GPUs," in Neural Networks (IJCNN), The 2010 International Joint Conference on, pp. 18, IEEE, 2010.

[27] A. Clauset, C. R. Shalizi, and M. E. J. Newman, "PowerLaw Distributions in Empirical Data," SIAM Review, vol. 51, pp. 661-703, Nov. 2009.

[28] A. Klaus, S. Yu, and D. Plenz, "Statistical analyses support power law distributions found in neuronal avalanches.," PloS one, vol. 6, p. e19779, Jan. 2011.

[29] T. J. Taylor, C. Hartley, P. L. Simon, I. Z. Kiss, and L. Berthouze, "Identification of criticality in neuronal avalanches: I. a theoretical investigation of the non-driven case," The Journal of Mathematical Neuroscience (JMN), vol. 3, no. 1, pp. 1-26, 2013.

[30] B. A. Cipra, "An introduction to the Ising model," American Mathematical Monthly, vol. 94, no. 10, pp. 937-959, 1987.

[31] M. Shanahan, "Metastable Chimera States in CommunityStructured Oscillator Networks," Chaos Woodbury Ny, vol. 20, no. 1, p. $013108,2010$. 